\section{Ask the expert: lung cancer}

Q1. I would like to know more about the practical role of autofluorescence bronchoscopy (AFB) in the early diagnosis of lung cancer lesions.

A. Mohan, New Delhi, India

A. Thanks for your question. The role of AFB is still somewhat controversial. With the new more sophisticated conventional white light bronchosopies, the role of fluorescence itself may be receding. Recent abstracts at the British Thoracic Society tended to suggest that, with modern equipment, an experienced bronchoscopist will identify as many abnormalities in the mucosa as they would with AFB. Having said that, there is no evidence yet that AFB has a place in routine bronchoscopy. It is being used as a surveillance tool in patients in whom routine bronchoscopy or follow-up bronchoscopy after resection identifies abnormal mucosa. Our policy at University College Hospital (UCH) is to repeat AFB every 4-6 months in individuals who have either severe dysplasia or carcinoma in situ (CIS). It is still controversial whether all CIS lesions become invasive. A recent study in Thorax (January 2007) showed that, while $50 \%$ of lesions became invasive, some remained stable and others disappeared. Other groups, e.g. SUTEDJA, recommend resection or treatment of all CIS lesions. Severe dysplasia or CIS probably represent a field cancerisation effect and these individuals are also likely to develop primary cancers in other distant parts of the lung.

In summary, I think severe dysplasia and CIS should be followed. If CIS becomes invasive, it is best treated locally with either photodynamic therapy or brachytherapy and not pulmonary resection as the lesion will be proximal and the resection large, i.e. lobectomy or pneumonectomy, and other primary lesions may follow in due course. However, there is no role for this procedure as a routine method of early detection. We happen to be running a national study in the UK looking at AFB as a means of detecting early lung cancer in smokers with mild or moderate COPD. The results of this will not be available for many years unfortunately. I hope this helps.

Q2. I would like to request your advice on the subject of the ease of smoking cessation by lung cancer patients. I have found many articles about smoking cessation in the prevention of lung cancer, or the evolution of lung cancer after cessation versus its evolution with persistent smoking, but how many lung cancer patients continue to smoke after the diagnosis of cancer, and are these patients more nicotine-dependent than other patients? Are there studies on the most effective smoking cessation treatments in these patients (e.g. high-dose nicotine-replacement therapy)? What advice do you give? In addition, it would be interesting to know whether there are any data on depression and smoking in lung cancer patients.

Many thanks for your answer.

G. Peiffer, Metz, France

A. Thanks for your question. I have not done a literature search for you on this question but, as you imply, there is considerable evidence that, even if current smokers develop lung cancer, up to $70 \%$ continue to smoke after diagnosis. This seems to apply to patients who undergo surgical resection as well as those who are inoperable. It is a lot to expect inoperable patients who are often symptomatic to both deal with their lung cancer and try to stop smoking. There seems to be too much going on to make them concentrate on smoking cessation and I think is understandable in view of their dreadful prognosis. I know of no studies looking at the most effective methods of employing smoking cessation in patients already diagnosed to have lung cancer. Personally, I do not try to hard to ask patients with advanced disease to stop smoking, but we certainly try to get patients who smoke and are fit for a resection to stop smoking before their resection. I hope this helps.
The new Breathe feature where we give you an expert and a topic, and you have the chance to ask them any questions you wish via breathe@ersj.org.uk

See page 234 for next month's expert and subjects.

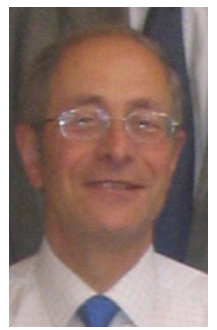

S. Spiro

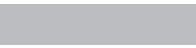

Department of Thoracic Medicine, University College Hospital, UCLH NHS Trust, Grafton Way, London, UK.

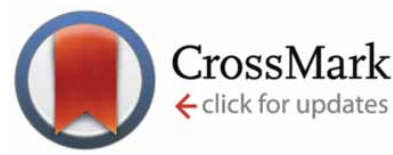

